\title{
ESTUDIO DE LA PRODUCTIVIDAD DE CORTE \\ EN MADERA Pinus elliottii \\ UTILIZANDO UN PROTOTIPO DE ASERRADERO PORTÁTIL
}

\author{
STUDY OF THE CUT PRODUCTIVITY IN WOOD Pinus elliottii \\ USING A PORTABLE SAWMILL PROTOTYPE
}

\author{
Washington Luiz Esteves Magalhães, Graciela I. Bolzon de Muniz' \\ María Guadalupe Lomelí Ramírez ${ }^{3}$, Djeison Cesar Batista ${ }^{3}$
}

\begin{abstract}
RESUMEN
El aserrío de trozas en tablas puede agregar valor a la madera producida en pequeñas propiedades rurales. El objetivo de este trabajo fue evaluar la calidad del aserrío de trozas de Pinus elliottii en un prototipo de aserradero portátil construido en cooperación entre una institución de investigación y una empresa privada. En base a los resultados obtenidos aún deberán realizarse algunos ajustes mecánicos en el prototipo. Sin embargo, el rendimiento encontrado fue superior al $71 \%$, la velocidad de alimentación estuvo entre 0,94 y $1,82 \mathrm{~m} / \mathrm{s}$, y la productividad máxima en madera aserrada fue de $2,17 \mathrm{~m}^{3} / \mathrm{h}$, que son valores compatibles o mejores que los modelos similares importados. La variación de espesor máximo quedó por debajo del límite máximo permitido por la norma Brasileña.
\end{abstract}

Palabras claves: aserradero portátil, calidad de corte, sierra cinta, Pinus elliottii.

\begin{abstract}
Sawmilling can add value to wood produced in small rural properties. The aim of this paper was to evaluate the quality of Pinus elliottii lumber saw-milled using the first prototype of portable sawmill made by the partnership among a research institution and industrial corporation. During saw-milling there were evaluated the volume yield, maximum productivity, cutting deviation, and feed speed of the prototype. The tests performed revealed that the prototype needs some mechanical modifications in order to decrease cutting deviation of the last saw-milled boards from the log. However, the volume yield was greater than $71 \%$, the feed speed of the prototype were between 0.94 and $1.82 \mathrm{~m} / \mathrm{s}$, and the maximum productivity was $2.17 \mathrm{~m}^{3} / \mathrm{h}$, these values are equal or better than those reached by similar imported equipments. The maximum cutting deviation was below the level allowed by the Brazilian standard NBR PB 1560.
\end{abstract}

Keywords: portable sawmill, cutting quality, band saw, Pinus elliottii.

\section{INTRODUCCIÓN}

Existen muchas ventajas documentadas de los aserraderos portátiles (Stewart y Hanson 1998; Eldred, 2000; Schaitza et al. 2000; Blackwell y Stewart, 2003; Smorfit et al. 2003; Becker et al. 2004; Venn et al. 2004; Bassani, 2005). El uso del aserradero portátil in situ tiene, entre tantas,

\footnotetext{
${ }^{1}$ Investigador de Embrapa Florestas, Colombo, PR. Brasil

${ }^{2}$ Profesor do Departamento de Engenharia e Tecnologia Florestal, UFPR. PR. Brasil.

${ }^{3}$ Estudiante de doctorado del Curso de Pós-graduação em Engenharia Florestal, UFRP. PR. Brasil.

${ }^{4}$ Prof. Departamento de Engenharia Florestal. Centro de Ciências Agrárias. Universidade Federal do Espírito

Santo. Brasil

Autor para correspondencia: glomeli@dmcyp.cucei.udg.mx

Recibido: 14.09.2009 Aceptado: 27.03.2010
} 
las siguientes ventajas: agregar valor a la madera para venta, tornar comercial una pequeña parcela forestal, producir madera para uso propio, reducir costos de transporte, producir una diversidad de productos tales como tablas, tablones, vigas, viguetas, listones, marcos, etc., evitando la necesidad de adquirirlos en el comercio, producir tamaños de piezas no estandarizadas. Así como también ayudar en la gestión del bosque y sus productos, permitir el aserrío de especies específicas y en pequeña escala, valorización de subproductos, como los residuos, tanto para uso propio como para venta.

Sin embargo, también existen desventajas como: baja productividad, actividad laboral intensa, esfuerzos por el levantamiento de peso a nivel del suelo. También, no siempre la calidad del producto final y el acabado son tan buenos como los que se pueden lograr en los aserraderos industriales. Además, el ambiente de trabajo puede no coincidir con las mejores prácticas industriales de salud ocupacional y seguridad.

Aunque existen muchas opciones de equipamiento de procedencia extrajera, hay una carencia de equipamientos nacionales para atender a los pequeños productores. Bassani (2005) desarrolló un proyecto de aserradero portátil para cortar trozas de hasta $70 \mathrm{~cm}$ de diámetro y tres metros de largo, aunque aún no se haya construido ningún prototipo.

Schaitza et al. (2000) mostraron las ventajas del uso de aserraderos portátiles por la reducción de gran parte de los costos de transporte de trozas, además de la eliminación del transporte de residuos tras el aserrío. Estos residuos generados en la operación del aserrío de trozas pueden ser vendidos a terceros o usados como sustrato para cultivo de champiñones (Kohari et al. 1997), como leña, o pueden ser transformados en carbón.

Los problemas que se pueden presentar durante el aserrío de trozas son alteraciones en las dimensiones de las piezas de madera pudiendo ser mayores que lo permitido por las normas. Los principales defectos de un aserrío incorrecto son el sobredimensionado, la variabilidad en el espesor de las tablas y la presencia de corteza.

El sobredimensionado es el exceso en espesor, ancho y/o largo, es por tanto lo dimensionado real menos la medida nominal. Aunque también puede ser referida a la tolerancia por defectos o irregularidades como la contracción. La variabilidad en el espesor de la tabla es referida a las desviaciones en el espesor o en el ancho de una misma pieza, ocasionada por el aserrío incorrecto. De acuerdo a la norma Brasileña TB 397 (Asociación Brasileña de Normas Técnicas, 1991a) la presencia de corteza en la tabla o canto muerto en una pieza de madera puede ser considerado un defecto.

Gatto et al. (2003) evaluaron la calidad de la madera aserrada con el uso de aserraderos fijos, de la región de la Cuarta Colonia de inmigración italiana, de Rio Grande do Sul, Brasil, y mostraron entre otros resultados, que es común una variación muy grande en espesores en las piezas aserradas y muchas veces superan el máximo permitido por la norma Brasileña.

Según Vital (2008) el rendimiento de aserraderos puede verse afectado por una serie de factores, incluido el nivel tecnológico de las máquinas utilizadas, el tipo de aserradero, el tipo de procesado de especies (coníferas o latifoliadas) y el diámetro de las trozas. Rocha (2002) menciona que otros factores como la calidad de los trozas, equipos, mano de obra utilizada y las técnicas de aserradero aplicadas afectan el rendimiento.

Murara et al. (2005) estudiaron el rendimiento de la madera de plantaciones de Pinus taeda en dos sistemas de aserradero, llamados convencional y optimizado, obtuvieron rendimientos promedios de $44,21 \%$ y $53,60 \%$ respectivamente. En ambos sistemas, las trozas se dividieron en cinco clases de diámetro y señalaron que el rendimiento aumenta en proporción al diámetro de las trozas en ambos sistemas estudiados. 
Biasi y Rocha (2003) estudiaron el rendimiento de madera aserrada de Pinus elliottii dividido en cuatro clases de diámetro $(8-18 \mathrm{~cm}, 14-24 \mathrm{~cm}, 20-32 \mathrm{~cm}$ y $33-45 \mathrm{~cm}$ ) y observaron un incremento de rendimiento con el aumento de las clases diamétricas

El trabajo realizado por Blackwell y Stewart (2003) hace una comparación entre modelos de aserraderos portátiles de los fabricantes Lucas Mill y WoodMizer. El modelo de Lucas Mill utiliza sierra circular como elemento cortante y el de la WoodMizer usa sierra cinta. De acuerdo a este estudio, la calidad de corte y el rendimiento de la madera aserrada empleando equipo con sierra cinta fue superior al de la sierra circular.

El objetivo de este trabajo fue evaluar el rendimiento y la productividad de aserrío de trozas de Pinus elliottii en un prototipo de aserradero portátil construido en cooperación entre una institución de investigación y una empresa privada brasileñas.

\section{MATERIAL Y MÉTODOS}

El aserradero portátil (figura 1) usado fue el primer prototipo construido por la EMBRAPA en cooperación entre FINEP (Financiadora de Estudos e Projetos), FUNPAR (Fundação da Universidade Federal do Paraná para o Desenvolvimento Técnico Científico) y la GIL Equipamientos Industriais Ltda. El aserradero trabaja con un motor a diesel de tipo estacionario de fabricación brasileña de marca Branco con $10 \mathrm{HP}$ de potencia nominal. Durante el aserrío la cinta fue enfriada y/o lubricada con agua con detergente doméstico. La lámina de la sierra cinta era nueva y tenía las siguientes características: espesor $1,1 \mathrm{~mm}$, ancho $32 \mathrm{~mm}$, largo de cuatro metros, ángulo libre de $10^{\circ}$, paso de los dientes 7/8", trabado de $0,3 \mathrm{~mm}$ y fue usada con el trabado y afilado que llegó del fabricante Woodmizer.

Fueron seleccionadas cuatro trozas de Pinus elliottii con diámetros próximos, sin defectos o tortuosidad. El diámetro promedio de las trozas fue de 25, 27, 32 y $34 \mathrm{~cm}$. y 2,6, 2,4, 2,6 y 3,10 m de largo, respectivamente. El volumen sin corteza de las trozas se calculó de acuerdo a la ecuación 1 , llamada ecuación de Huber, a continuación.

$$
v=\frac{\left(\pi \times d^{2}\right)}{40000} \times 1
$$

Dónde:

$\mathrm{v}$ : volumen de la troza $\left(\mathrm{m}^{3}\right)$;

d: diámetro de la mitad del largo de la troza $(\mathrm{cm})$; sin corteza

1: largo de la troza $(\mathrm{m})$.

La experiencia con los aserraderos móviles que usan sierra cinta del mismo tipo de la usada en este trabajo, muestran que cuatro es el número de trozas para ser aserradas antes de cambiarse la cinta o someterla a un nuevo trabamiento y afilado. Algunas veces es necesario cambiar la cinta después de la primera troza. Eso depende de cuanta arena o piedras están adheridas en la troza, ya que por regla general, los pequeños productores derrumban los árboles con moto-sierra y arrastran las trozas con tractores a cierta distancia. Además que la operación de descortezado casi nunca es realizada por los pequeños productores. 


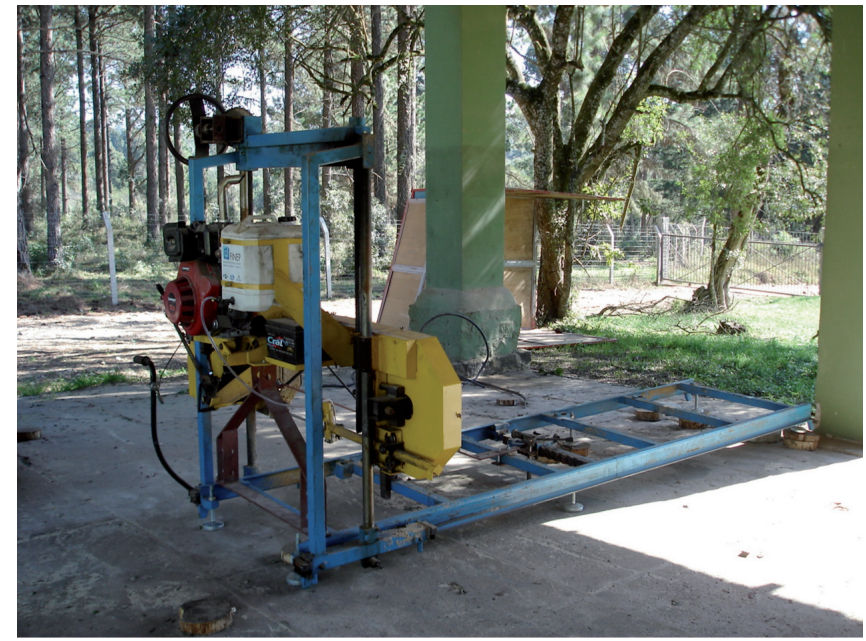

Figura 1. Foto del primer prototipo de aserradero portátil construido en cooperación entre Embrapa/FINEP/FUNPAR/Gil Equipamentos Industriais Ltda.

Para reforzar esta experiencia se contactó con el fabricante de sierras cintas Starret que suministra las sierras para algunos aserraderos industriales, en la región de Ponta Grossa, Paraná, Brasil. El cuerpo técnico de esa empresa informó que el tiempo de vida útil de sus cintas en servicio es aproximadamente de 20 horas. Mientras que las industrias usan generalmente el siguiente procedimiento: retirar la cinta, trabar y afilar cada 4 horas de aserrío. Ésta práctica de la industria concordó con la experiencia que se adquirió en EMBRAPA para el caso de los aserraderos móviles importados Woodmizer y Husqvarna. En caso que los trabajos de aserrío prosigan sin un cambio de sierra cinta, la velocidad de alimentación puede llegar a disminuir a la mitad, además la variación del espesor y la calidad de la superficie de las tablas aserradas quedan seriamente comprometidas. Aunque también depende de la calidad de la lámina de la sierra cinta, de la especie de madera, etc.

El aserrío de la troza fue realizado retirando primero las costeras para la formación de una basa. Luego fueron cortadas tablas como se observa en la figura 2. Estas tablas fueron aserradas con un espesor entre 26 y $27 \mathrm{~mm}$ para que después del secado y cepillado el espesor final fuese de $24 \mathrm{~mm}$. Sin embargo, fueron obtenidos espesores diferentes durante la eliminación de las costeras de las trozas cónicas y torcidas. Este procedimiento de corte es el comúnmente utilizado por los fabricantes internacionales para medir la productividad de sus máquinas.

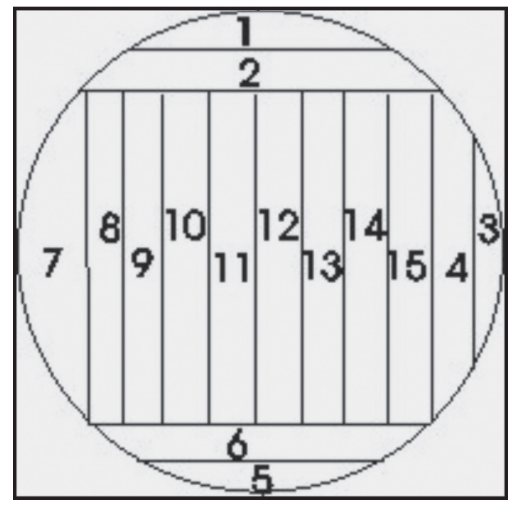

Figura 2. Diagrama de aserrío de las trozas 
La velocidad de alimentación fue calculada tomando en cuenta el largo de la troza dividido por el tiempo usado para que la lámina de la sierra cinta efectúe el corte longitudinal de la troza. El tiempo de la velocidad de avance fue medido con precisión de segundos con el uso de un cronómetro y el largo de la troza fue medido con una cinta métrica.

No existe norma brasileña para la medición del espesor, fue utilizado un procedimiento modificado reportado por Blackwell y Stewart, (2003). El control del espesor de la tabla a lo largo del corte fue realizado tomando medidas de espesor cada $20 \mathrm{~cm}$ a lo largo de la pieza de madera, utilizando un vernier con precisión de $0,1 \mathrm{~mm}$.

\section{RESULTADOS Y DISCUSIÓN}

En la Tabla 1 se muestran los resultados de las velocidades de alimentación calculadas a la lámina de sierra cinta durante el aserrío de las trozas de Pinus elliottii. Durante el aserrío fueron producidos cuatro costados por troza y varias tablas, siendo que algunas de ellas presentaban restos de corteza.

Tabla 1. Velocidad de alimentación en el aserrío de trozas de Pinus elliottii

\begin{tabular}{|c|c|c|c|c|c|c|}
\hline TROZA & $\begin{array}{l}\text { Productos del } \\
\text { aserrío }\end{array}$ & $\begin{array}{l}\text { Ancho } \\
\text { de la } \\
\text { tabla } \\
(\mathrm{cm})\end{array}$ & $\begin{array}{l}\text { Velocidad de } \\
\text { alimentación } \\
\text { promedio } \\
(\mathrm{m} / \mathrm{s})\end{array}$ & $\begin{array}{l}\text { Desviación } \\
\text { estándar } \\
\text { de la } \\
\text { velocidad de } \\
\text { alimentación }\end{array}$ & $\begin{array}{c}\text { Velocidad } \\
\text { máxima de } \\
\text { alimentación } \\
(\mathrm{m} / \mathrm{s})\end{array}$ & $\begin{array}{c}\text { Velocidad } \\
\text { minima de } \\
\text { alimentación } \\
(\mathrm{m} / \mathrm{s})\end{array}$ \\
\hline \multirow[b]{2}{*}{1} & costados & \multirow[b]{2}{*}{23} & 0,140 & 0,04 & 0,172 & 0,094 \\
\hline & $\begin{array}{c}\text { Tabla con corteza } \\
\text { Tabla }\end{array}$ & & $\begin{array}{l}0,126 \\
0,153 \\
\end{array}$ & $\begin{array}{l}0,00 \\
0,04\end{array}$ & $\begin{array}{l}0,129 \\
0,182 \\
\end{array}$ & $\begin{array}{l}0,124 \\
0,097 \\
\end{array}$ \\
\hline \multirow[b]{2}{*}{2} & costados & \multirow[b]{2}{*}{23} & 0,125 & 0,06 & 0,174 & 0,065 \\
\hline & $\begin{array}{c}\text { Tabla con corteza } \\
\text { Tabla } \\
\end{array}$ & & $\begin{array}{l}0,103 \\
0,131 \\
\end{array}$ & $\begin{array}{l}0,03 \\
0,03 \\
\end{array}$ & $\begin{array}{l}0,131 \\
0,174 \\
\end{array}$ & $\begin{array}{l}0,065 \\
0,087 \\
\end{array}$ \\
\hline \multirow[b]{2}{*}{3} & costados & \multirow{3}{*}{21} & 0,118 & 0,03 & 0,182 & 0,124 \\
\hline & $\begin{array}{c}\text { Tabla con corteza } \\
\text { Tabla }\end{array}$ & & $\begin{array}{l}0,111 \\
0,120\end{array}$ & $\begin{array}{l}0,01 \\
0,01\end{array}$ & $\begin{array}{l}0,129 \\
0,129\end{array}$ & $\begin{array}{l}0,103 \\
0,129\end{array}$ \\
\hline \multirow{3}{*}{4} & costados & & 0,133 & 0,02 & 0,163 & 0,124 \\
\hline & Tabla con corteza & 19 & 0,123 & 0,00 & 0,123 & 0,129 \\
\hline & Tabla & & 0,120 & 0,02 & 0,136 & 0,124 \\
\hline
\end{tabular}

Las velocidades de alimentación no fueron uniformes ya que el cabezal del equipo es empujado manualmente sobre el carril. La velocidad que para producir una superficie con un mejor acabado o una menor ondulación no pudo ser definida en este experimento.

Esas velocidades de alimentación fueron casi $70 \%$ superiores a las de los aserraderos modelo de Lucas Mill con sierra circular y de 30\% superiores al modelo de Woodmizer con sierra tipo cinta de acuerdo a lo reportado por Blackwell y Stewart (2003). En el caso del equipo Lucas Mill, la gran diferencia se debe principalmente a los diferentes elementos de corte. Para el caso del Woodmizer, la diferencia puede ser atribuida a las diferentes maderas usadas. La comparación permite mostrar que el prototipo brasileño tiene condiciones de substituir los equipos importados.

En la Tabla 2 se presentan las dimensiones de las tablas obtenidas durante el aserrío de la troza 1 de Pinus con un rendimiento de $68 \%$. 
Tabla 2. Dimensiones de las tablas aserradas de la troza 1

\begin{tabular}{cccc}
\hline Tablas & Ancho $(\mathrm{cm})$ & Espesor promedio $(\mathrm{mm})$ & Volumen $\left(\mathrm{m}^{3}\right)$ \\
\hline 1 & 23 & 28,2 & 0,0201 \\
2 & 23 & 29,6 & 0,0211 \\
3 & 23 & 26,8 & 0,0191 \\
4 & 23 & 26,6 & 0,0190 \\
5 & 23 & 26,8 & 0,0191 \\
6 & 23 & 26,5 & 0,0189 \\
7 & 23 & 21,9 & 0,0156 \\
8 & 23 & 31,9 & 0,0227 \\
R9 & 14 & 28,5 & 0,0124 \\
R10 & 12 & 28,5 & 0,0106 \\
R11 & 16 & 25,9 & 0,0128 \\
\hline TOTAL & & & 0,1914
\end{tabular}

Obs.: Tablas con iniciales $\mathrm{R}$ se refieren a tablas con corteza, su ancho fue medido después del canteado

Las tablas con corteza fueron codificadas como R9, R10, R11, el ancho de esas tablas fue medido después del canteado. Los valores son menores que el resto de tablas obtenidas, porque son las más externas de la troza y fueron retiradas para formar el bloque. El volumen total de tablas producidas durante el aserrío fue de $0,191 \mathrm{~m}^{3}$, siendo incluida en este cálculo las tablas con corteza después del canteado. Por tanto, el rendimiento de madera aserrada obtenida para la troza 1 fue de $68 \%$.

El tiempo total de corte para la troza 1, o sea, la suma de los tiempos de alimentación durante el corte de cada costado o tabla, fue de 318 segundos (Tabla 3). Se puede calcular, que la producción máxima posible del equipo sería de $2,17 \mathrm{~m}^{3} /$ hora, que equivale a una producción de $17,33 \mathrm{~m}^{3}$ cada 8 horas de trabajo. Sin embargo, cabe resaltar que este valor no se refiere al valor práctico en que los productores se deben basar, en virtud de no tener en cuenta los tiempos para carguío y rotación de la troza en aserradero.

La Tabla 3 muestra las dimensiones de las trozas, el tiempo utilizado en los cortes de las tablas, el volumen de tablas aserradas, el rendimiento y la productividad máxima del aserrío.

Con una producción máxima teórica media de $1,80 \mathrm{~m}^{3} / \mathrm{h}$ para cuatro trozas, para una jornada de 8 h/día de trabajo resulta una producción de $14,4 \mathrm{~m}^{3} /$ día.

Tabla 3. Dimensiones de trozas, tiempo de aserrío, volumen aserrado y rendimiento

\begin{tabular}{|c|c|c|c|c|c|c|}
\hline TROZA & $\begin{array}{l}\text { Diámetro } \\
\text { medio } \\
(\mathrm{cm})\end{array}$ & $\begin{array}{l}\text { Largo } \\
(\mathrm{m})\end{array}$ & $\begin{array}{l}\text { Volumen } \\
\left(\mathrm{m}^{3}\right)\end{array}$ & $\begin{array}{c}\text { Tiempo } \\
\text { utilizado solo } \\
\text { en los cortes } \\
\text { (s) }\end{array}$ & $\begin{array}{l}\text { Volumen de } \\
\text { tablas aserradas } \\
\left(\mathrm{m}^{3}\right)\end{array}$ & $\begin{array}{c}\text { Rendimiento } \\
(\%)\end{array}$ \\
\hline 1 & 34 & 3,1 & 0,2814 & 318 & 0,1914 & 68 \\
\hline 2 & 32 & 2,6 & 0,2091 & 333 & 0,1689 & 81 \\
\hline 3 & 25 & 2,6 & 0,1374 & 230 & 0,0929 & 68 \\
\hline 4 & 27 & 2,4 & 0,1276 & 198 & 0,0874 & 68 \\
\hline Total & & & 0,7556 & - & 0,5406 & 71 \\
\hline \multicolumn{7}{|c|}{ Rendimiento medio (\%) } \\
\hline \multicolumn{4}{|c|}{ Tiempo total utilizado solo en los cortes (s) } & 1079 & & \\
\hline \multicolumn{4}{|c|}{ Producción máxima teórica } & & & \\
\hline
\end{tabular}


La producción máxima teórica cayó de 2,17 en el aserrío de la troza 1 a 1,80 m³/hora en la media de las cuatro trozas, debido a factores tales, como la pérdida de filo, al trabamiento de los dientes de la lámina de corte y también la clase diamétrica de las trozas. Aunque la velocidad de alimentación disminuye con el aumento del diámetro de la troza, éste efecto es contrarrestado por el mayor volumen de madera aserrada. Cuanto mayor sea la clase de diámetro, mayor será el ancho de las tablas producidas resultando por tanto un mayor volumen de madera. Sin embargo, si la longitud de la troza es muy corta, se pierde mucho tiempo para reposicionar la lámina y también para colocación de otra troza. El plano de corte influye en el rendimiento por la geometría que limita la máxima cantidad de madera útil factible de ser aserrada. Un plano de corte muy sofisticado aumenta también el tiempo para reposicionar la troza, lo que ocasiona pérdida de productividad. Trozas con tortuosidad o conicidad excesiva también contribuyen para una disminución en el aprovechamiento de madera por troza. En este trabajo, la razón volumen de madera/tiempo aumentó a pesar de aumentar el tiempo para el aserrío con el incremento de la clase diamétrica (figura 3).

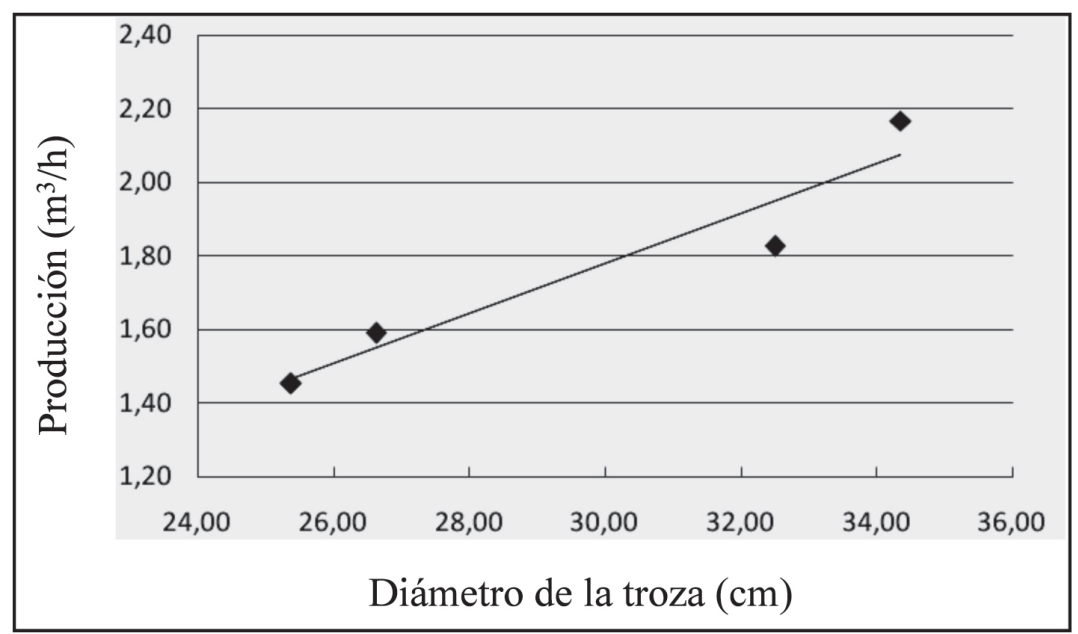

Figura 3. Producción volumetrica de madera aserrada versus diametro de la troza.

Para mayor claridad y facilidad de análisis se presenta en la figura 4 la variación de los espesores a los largo de las tablas de la troza 1 sin presencia de corteza en sus costados.

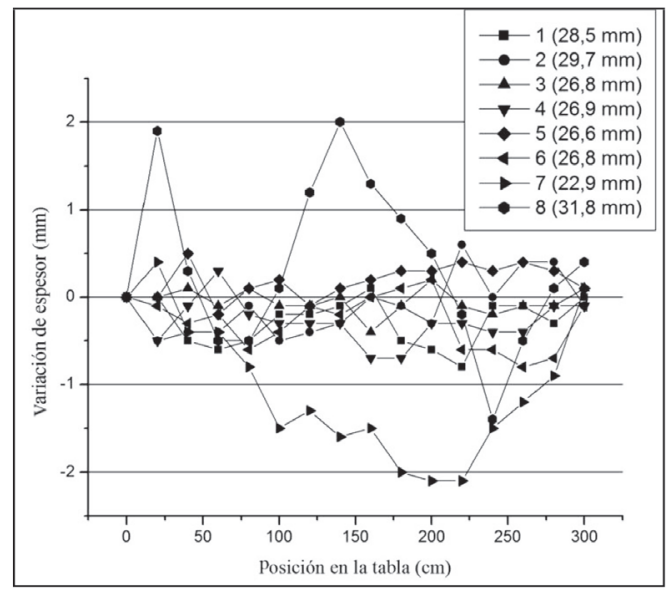

Figura 4. Espesores de las tablas de la troza 1. 
A excepción de la tablas 7 y 8 , todas las demás piezas de la troza 1 presentaron variaciones que oscilaron entre 0,6 y - $-0,8 \mathrm{~mm}$, o sea una variación máxima de $1,4 \mathrm{~mm}$ entre tablas, siendo que cada tabla separadamente presentó una variación no mayor de $0,8 \mathrm{~mm}$ (Figura 4). Aun cuando, la especie usada en este estudio fue diferente de las evaluadas por Blackwell y Stewart (2003), estos resultados son mejores que los presentados para los aserraderos de las compañías WoodMizer y Lucas Mill y en general se consideran buenos resultados para un aserradero portátil.

En la Tabla 4, se presentan los resultados del espesor promedio, el mayor y el menor valor de los espesores, la mayor diferencia entre éstos (variación de espesores máximos) y la desviación estándar de los espesores de las tablas de la troza 1.

Tabla 4. Medidas de las tablas de la troza No. 1

\begin{tabular}{lcccccccc}
\hline \multicolumn{1}{c}{ Tablas } & 1 & 2 & 3 & 4 & 5 & 6 & 7 & 8 \\
\hline Espesor medio (mm) & 28,2 & 29,6 & 26,8 & 26,6 & 26,8 & 26,5 & 21,9 & 31,9 \\
Espesor máximo (mm) & 28,6 & 30,3 & 27,0 & 27,2 & 27,1 & 27,0 & 23,3 & 33,5 \\
$\begin{array}{l}\text { Espesor mínimo (mm) } \\
\text { Variación del espesor }\end{array}$ & 27,7 & 29,2 & 26,4 & 26,2 & 26,4 & 26,0 & 20,8 & 30,1 \\
máximo (mm) & 0,9 & 1,1 & 0,6 & 1,0 & 0,7 & 1,0 & 2,5 & 3,4 \\
$\begin{array}{l}\text { Desviación estándar de } \\
\text { los espesores }\end{array}$ & 0,27 & 0,34 & 0,14 & 0,25 & 0,19 & 0,31 & 0,80 & 0,93 \\
\hline
\end{tabular}

Exceptuando las tablas 7 y 8 , la mayor variación de espesor fue de 1,1 $\mathrm{mm}$ para la tabla número 2 y el menor fue de $0,6 \mathrm{~mm}$ para la número 3 . Esos valores son considerados como normales y están dentro de la tolerancia según la norma PB-1560 (Asociación Brasileña de Normas Técnicas, 1991c), en que el límite máximo de variación es de $2 \mathrm{~mm}$. La misma norma permite que $25 \%$ del lote presente una variación de su espesor de 3 y $4 \mathrm{~mm}$ para piezas con espesores nominales en los rangos $12-25 \mathrm{~mm}$ y $32-50 \mathrm{~mm}$, respectivamente. Es importante recordar que para efecto de clasificación de las tablas según la estándar CB 205 (Asociación Brasileña de Normas Técnicas, 1991b) las categorías súper, extra, primera y segunda clase no son permitidas sobremedidas en el espesor de $2 \mathrm{~mm}$.

La figura 5 muestra la variación del espesor medido para todas las tablas cortadas en cada troza. El mismo problema señalado para la primera troza ocurrió en todas las otras trozas aserradas.

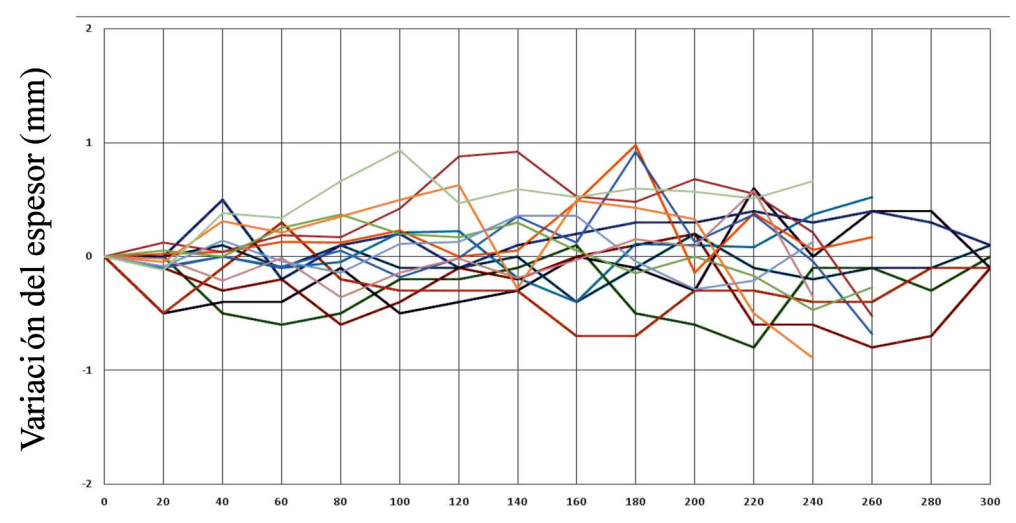

Posición en la tabla $(\mathrm{cm})$

Figura 5.Variación de espesor de las tablas de las trozas 1, 2, 3 y 4 
Como puede observarse en la figura 5 las variaciones de los espesores de las tablas quedan por debajo de los límites establecidos en la norma brasileña. La variación máxima encontrada fue de $1,52 \mathrm{~mm}$ y la mínima fue de $0,60 \mathrm{~mm}$. De un modo general, los valores más malos para las variaciones en los espesores son encontrados para las últimas trozas aserradas con la misma lámina. Ese hecho puede ser explicado tanto por el desgaste de la lámina con la pérdida del filo y del des-trabamiento de los dientes de corte o debido a falla humana por el cansancio que se produce después de varias horas de trabajo. En este caso, la tensión de la cinta no fue ajustada constantemente como en el caso de las máquinas Woodmizer. Pero hubo ajuste de las guías para cada diámetro de troza.

\section{CONCLUSIONES}

El primer prototipo de aserradero portátil construido en cooperación entre la institución de investigación y la empresa privada, presentó rendimiento superior al 66\% y productividad teórica máxima de 2,17 m³/hora para trozas de Pinus elliottii con diámetro medio menor de $34 \mathrm{~cm}$ y $3,10 \mathrm{~m}$ de largo;

La velocidad de alimentación no fue uniforme debido a la operación de aserrío que fue ejecutada de forma manual y depende de la experiencia del operador;

El equipo mantuvo una variación de espesor debajo del máximo permitido por la estándar Brasileña NBR PB 1560 y posibilita que las tablas reciban una mejor clasificación en relación a este defecto.

Se puede promover un mejoramiento mecánico en el equipamiento para disminuir defectos de aserrado durante el corte.

Nuevos experimentos deben ser conducidos para evaluar la influencia del grado de tensión y velocidad de alimentación en la calidad de acabado y variación de espesores de las tablas aserradas.

\section{REFERENCIAS}

Associação Brasileira de Normas Técnicas. 1991a. madeira serrada de coníferas provenientes de reflorestamento, para uso geral: terminologia. TB 397. Rio de Janeiro.

Associação Brasileira de Normas Técnicas. 1991b. madeira serrada de coníferas provenientes de reflorestamento, para uso geral: classificação. CB 205. Rio de Janeiro,.

Associação Brasileira de Normas Técnicas. 1991c. Madeira serrada de coníferas provenientes de reflorestamento, para uso geral: dimensões e lotes: padronização. PB 1560. Rio de Janeiro..

Bassani, W. 2005. Projeto de uma serraria móvel. Monografia (Graduação) - Faculdade de Engenharia e Arquitetura, Engenharia Mecânica, Universidade de Passo Fundo, Passo Fundo.

Becker, D. R.; Hjerpe, E. E.; Lowell, E. C. 2004. Economic assessment of using a mobile micromill for processing small-diameter ponderosa pine. Portland: USDA, Forest Service, Pacific Northwest Research Station (USDA. For. Serv. Gen. Tech. Rep. PNW-GTR-623). 40 p.

Biasi, C.P.; Rocha, M.P. 2003. Rendimento em madeira serrada de Pinus elliottii. In: Congresso Florestal Brasileiro, 8, 2003, São Paulo. Anais. São Paulo: [s.n.]. 
Blackwell, P.; Stewart, M. 2003. Using portable sawmills to produce high value timber from farm trees in the semi-arid zone. (RIRDC publication, 03/046). RIRDC Project n. PN99.2001. A report for the RIRDC/Land \& Water Australia/FWPRDC/MDBC Joint Venture Agroforestry Program and the Natural Heritage Trust. 141 p. Consultado 2 ago. 2007. Disponible en: http://www.rirdc.gov.au/ reports/AFT/03-046.pdf.

Eldred, L. 2000. On site sawmilling and timber conversion: information pack. Oxon: The Chilterns, Chiltern Woodlands Project. Consultado 2 ago. 2007.. Disponble en::

http://www.chilternsaonb.org/downloads/publications/On_site_Sawmilling.pdf.

Gatto, D. A.; Santini, E. J.; Haselein, C. R.; Durlo, M. A. 2003. Qualidade da madeira serrada na região da quarta colônia de imigração italiana do Rio Grande do Sul. Ciência Florestal 14 (1): 223233.

Kohari, E. K.; Amazonas, M.A.L. de A.; Carvalho, F.J.P. de C. 1997. Potencial de crescimento micelial do fungo Pleurotus sajor-caju em serragem e casca de Pinus spp. e resíduo de infusão de erva-mate. In: Workshop sul-americano sobre usos alternativos de resíduos de origem florestal e urbana. Curitiba. Anais. Colombo: Embrapa Florestas, p. 150-155.

Murara Junior, M.; Rocha, M.P.; Timofeiczyk Junior, R. 2005. Rendimento em madeira serrada de Pinus taeda para duas metodologias de desdobro. Floresta 35 (3): 473-483.

Rocha, M.P. 2002. Técnicas e planejamento de serrarias. Série didática nº2/01.Fupef, Curitiba. 121 p.

Schaitza, E. G.; Hoeflich, V. A.; Rodigheri, H.; Ferron, R. 2000. A utilização de serrarias portáteis em florestas de pínus e eucaliptos em pequenas propriedades rurais: A experiência da Embrapa/Cotrel. Colombo: Embrapa Florestas. (Embrapa Florestas. Circular técnica, 35). 25 p.

Smorfit, D.; Harrison, S.; Herbohn, J. 2003. Portable sawmills in a high-value rainforest cabinet timber industry in North Queensland. Small-scale. Forest Economics, Management and Policy 2 (1): 21-36.

Stewart, M.; Hanson, I. 1998. On-site processing for farm forestry: does it stack up? Barton: Rural Industries Research \& Development Corporation, (RIRDC short report, n. 38). Consultado 2 ago. 2007. Disponible en:

file://C:/Documents\%20and\%20Settings/ainfo/Meus\%20documentos/Comite_de_Publicacoes_ Referencias/ARTIGOS\%20SERRARIA/on\%20site $\% 20$ processing $\% 20$ for $\% 20$ farm $\% 20$ forestry. htm.

Venn, T. J.; Mcgavin, R. L.; Leggate, W. W. 2004. Costs of portable sawmilling timbers from the acacia woodlands of Western Queensland, Australia. Small-scale Forest Economics, Management and Policy 3 (2): 161-175.

Vital, B.R. 2008. Planejamento e operação de serrarias. Viçosa, MG, Editora UFV. 211 p. 